\title{
Trypanosome variable surface glycoproteins: composite genes and order of expression
}

\author{
Genevieve Thon, ${ }^{1}$ Theo Baltz, ${ }^{2}$ Christiane Giroud, ${ }^{2}$ and Harvey Eisen ${ }^{1}$ \\ ${ }^{1}$ Fred Hutchinson Cancer Research Center, Seattle, Washington 98104 USA; ${ }^{2}$ Universite de Bordeaux II, Immunologie et \\ Biologie Parasitaire, 33076 Bordeaux Cedex, France
}

\begin{abstract}
Combinatorial processes increase the diversity of variable surface glycoproteins (VSGs) expressed by Trypanosoma equiperdum. We show here that a single telomeric pseudogene provides the 3 ' portion of three distinct $T$. equiperdum VSG genes by recombination with different $5^{\prime}$ donor pseudogenes. Regions of sequence homology among the pseudogenes determine the sites of recombination in the formation of the expressed copies. This suggests that the recombination between any given basic copy $(\mathrm{BC})$ and the expression-linked copy (ELC) depends on their sharing homology. We present evidence that this is the case and propose that such rules account for the order of expression of the VSGs. These results demonstrate how homologous recombination can generate an ordered sequence of gene expression.
\end{abstract}

[Key Words: Trypanosoma equiperdum; antigenic variation; surface antigen; gene conversion; recombination; pseudogene]

Received March 28, 1990; revised version accepted May 30, 1990.

African trypanosomes express large repertoires of variable surface glycoproteins (VSGs). Periodic replacements of the VSG allow the survival of the parasites in the bloodstream of the host. Capbern et al. (1977) showed that at least 100 different antigens of Trypanosoma equiperdum appear in a quasisequential manner during rabbit infections.

VSG genes from $T$. equiperdum and from the related species Trypanosoma brucei are transcribed from specific telomeric loci or expression sites (for review, see Pays and Steinert 1988). A process similar to gene conversion leads to successive replacements of the expressed VSG gene in an expression site. Silent genes, or basic copies (BCs), act as donors in that process, which probably requires a $5^{\prime}$ and $3^{\prime}$ sequence homology between the $\mathrm{BC}$ and the expression site. On the $5^{\prime}$ side of the $\mathrm{BC}$, homology is provided either by imperfect 76-bp repeats present in large numbers in the expression sites (Liu et al. 1983; Campbell et al. 1984; Florent et al. 1987) or by other sequences related to the ones occupying the expression site at the time (Donelson et al. 1983; Pays et al. 1983; Lee and Van der Ploeg 1987). On the $3^{\prime}$ side of the $\mathrm{BC}$, the recombination may occur within telomeric sequences, near short highly conserved blocks found $3^{\prime}$ of the VSG-coding sequences (De Lange et al. 1983; Michels et al. 1983), in conserved coding regions at the very $3^{\prime}$ end of VSG genes (Bernards et al. 1981; Michels et al. 1983), or in short sequences in the coding region shared by a BC and the VSG gene occupying the expression site (Roth et al. 1989; Thon et al. 1989).

Longacre and Eisen (1986) presented evidence that ex- pression-linked copies (ELCs) expressed early in T. equiperdum infections are derived from complete BC genes, whereas ELCs expressed later are formed by duplication and combination of several BCs. These results suggested a model for how the order of expression of the VSGs may be determined; this is illustrated in Figure 1. Some silent BCs code for functional VSGs and display two regions of homology with the expression sites, one located $5^{\prime}$ of the coding region and one located $3^{\prime}$ of it. These BCs can enter an expression site regardless of the sequence present in it. Therefore, they tend to transpose often and are expressed early during an infection. Some BCs, on the other hand, miss one of the regions of homology or do not have a complete VSG open reading frame. Such BCs cannot be inserted into an expression site unless they have internal homology with the resident VSG gene. Families of incomplete BCs may share the same internal sequence similarity with a given ELC. The probability of their being transposed into an expression site is not constant. When the expression site is occupied by a nonhomologous VSG gene, the probability of transposition is low, but when a gene with which they share homology occupies the expression site, the probability of their transposition increases dramatically. As a consequence, families of VSGs would tend to be expressed at about the same time during an infection. One BC could share different sequence similarities with several other BCs as well. This could impose an order on the expression of the families relative to each other.

As a first step in testing the model presented above, two late-expressed VSG genes were characterized in de- 
Early

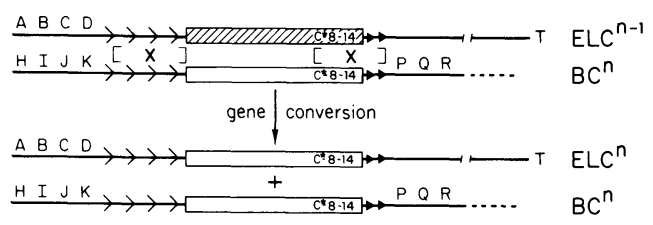

Middle and Late

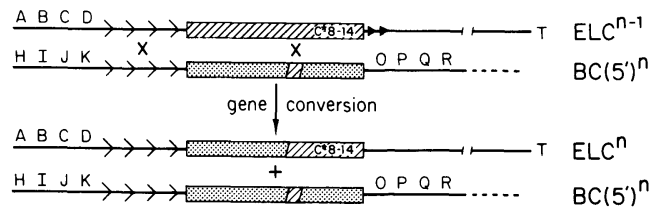

Figure 1. Model for the order of expression of the VSGs. The flanking regions of early-expressed VSG $B C s\left(B C^{n}\right)$ have some sequence homology with the expression sites, allowing transposition to occur independently of the gene occupying the expression site. Early BCs also have complete open reading frames. In contrast, late-expressed $\mathrm{BCs}\left[\mathrm{BC}\left(5^{\prime}\right)^{n}\right]$ do not encode complete VSGs or miss the homology with the expression sites. Therefore, their expression is dependent on a recombination with the VSG gene present in the expression site at the time. Elements conserved $3^{\prime}$ of VSG-coding regions are indicated. $\left(C^{\star}\right)$ Region rich in cytosine; (8) TGCTACTT; (14) TGATATATTTTAAC. Large arrows represent sequences common to the $5^{\prime}$ regions of $\mathrm{BCs}$ and expression sites; small arrows represent sequences common to the $3^{\prime}$ regions of BCs and expression sites. (T) Telomere.

tail. Three or four BCs are involved in the formation of the gene that encodes T. equiperdum VSG-78 (Roth et al. 1986, 1989) and three BCs participate in the formation of the VSG-20 gene (Thon et al. 1989). Two 5' donor peudogenes, VSG-20 BC1 and VSG-20 BC2, form a mosaic that encodes most of VSG-20. The mosaic is joined to a $3^{\prime}$ donor pseudogene, VSG-20 BC3, which encodes 51 amino acids at the carboxyl terminus of the protein. Homology between the VSG-20 $3^{\prime}$ and 5' donors is limited to an 85 -bp segment within which the recombination occurs.

We investigated whether $5^{\prime}$ donors different from the VSG-20 5' donors could be expressed in combination with the VSG-20 3' donor. Here, we present the characterization of two such $5^{\prime}$ donors that are used in two different antigenic variants of $T$. equiperdum, Bordeaux Trypanozoon antigenic type $20^{*}($ BoTat-20*) and BoTat20bis. The characterization of these variants and their BCs allowed us to test a prediction of our model for the order of VSG gene expression. The model predicts that early variants should appear with an equal probability in populations derived from any cloned variant, whereas late variants should appear preferentially within a population that expresses an appropriate gene in the expression site. We compared the sequences of the ELC and BCs used in the two newly characterized variants with the VSG-20 gene and with other VSG genes. Some of these sequences present localized similarities. The model predicts that some switches should therefore be more likely to occur than others in some trypanosome clones. First, we tested whether these switches occurred and in which variants they occurred; second, whether switches to other late variants could be detected; and third, whether switches to an early variant, BoTat-1, could be detected.

\section{Results}

A VSG encoded by a combination of the VSG-20 $3^{\prime}$ donor and a gene different from the VSG-20 5' donors might be expected to share some, but not all, epitopes with VSG-20. We used a rabbit polyclonal antiserum raised against the purified VSG-20 (Capbern et al. 1977) to identify trypanosome clones immunologically related to BoTat-20. In addition to this polyclonal serum, we raised six hybridomas producing anti-VSG-20 antibodies. These monoclonal antibodies distinguish between BoTat-20 and two other clones immunologically related to BoTat-20, which were obtained as described below.

\section{Isolation of BoTat-20bis}

A rabbit was infected with T. equiperdum clone BoTat1. Blood samples were taken every third day after infection, and the presence of trypanosomes related to BoTat20 was assayed for by immunofluorescence after propagation of the trypanosomes in a mouse and fixation on slides. Variants reactive with the anti-VSG-20 polyclonal serum were detected at day 30 . A clone of such a variant, BoTat-20bis, was obtained, and its reactivity with several anti-VSG-20 monoclonal antibodies was assayed. BoTat-20bis does not react with any of the six anti-VSG-20 monoclonal antibodies.

\section{Isolation of BoTat-20*}

A mouse was infected with the previously characterized clone BoTat-20, and ascites fluid containing anti-VSG20 monoclonal antibody $\mathrm{E}_{20} \mathrm{H}_{16}$ was injected at the same time. A variant, BoTat $-20^{\star}$, was isolated from this mouse and clonally expanded. BoTat $-20^{\star}$ still reacts with the anti-VSG-20 polyclonal serum after fixation on slides but not with the monoclonal antibody $\mathrm{E}_{20} \mathrm{H}_{16}$ nor with any of the other anti-VSG-20 monoclonal antibodies that we made.

\section{BoTat-20, BoTat-20bis, and BoTat-20* appear to the host as different variants}

Although BoTat-20* and BoTat-20bis were selected by reactivity to an anti-VSG polyclonal serum, the absence of reactivity of VSG-20bis and VSG-20* with all of the anti-VSG-20 monoclonal antibodies suggested that the two proteins were only distantly related to VSG-20. We investigated the possibility that on live trypanosomes, the epitopes common to the three VSGs are not accessible to the host immune system. Mice were immunized with killed trypanosomes of serotype $20,20 \mathrm{bis}$, or $20^{*}$ and subsequently challenged with live BoTat-20, BoTat20 bis, or BoTat-20* trypanosomes. This experiment, presented in Table 1 , shows that the epitopes common 
to the three proteins are not sufficient to induce cross immunization in vivo. Three mice died after immunization and challenge by BoTat-20. At the time of their death, they were infected by BoTat-1 rather than BoTat20 . This is the consequence of a switch that occurred from the expression of VSG-20 to the expression of VSG-1, for which we present additional evidence below. All of the other mice that died, did so from infection by the trypanosome serotype used for the challenge.

\section{VSG-20bis and VSG-20* gene families}

To determine whether the genes for VSG-20, VSG-20bis and VSG- $20^{*}$ had related sequences, we prepared cDNA clones coding for VSG-20bis and VSG-20*, which were used as probes on Northern and Southern blots (Fig. 2).

The hybridization patterns on Northern blots indicate that the $3^{\prime}$ ends of BoTat-20, BoTat-20bis, and BoTat$20^{*}$ mRNAs have related sequences, whereas their $5^{\prime}$ ends do not cross-hybridize. This suggests that the common epitopes that react with the polyclonal serum are located in the carboxy-terminal region of the three VSGs.

Both VSG-20bis and VSG-20* have been activated by duplicative transpositions. The VSG-20* ELC is in the same expression site, as was VSG-20 ELC (data not shown). The $3^{\prime}$ ends of BoTat-20bis and BoTat-20* cDNAs hybridize under stringent conditions to only one locus in BoTat-1 DNA, characterized previously as VSG-20 BC3 (Thon et al. 1989). This locus is closely linked to a telomere. Thus, the ELCs of BoTat-20, BoTat-20bis and BoTat-20* use the same $3^{\prime}$ donor gene. As shown in Figure 2B, a cDNA probe specific for the $5^{\prime}$ end of VSG-20bis mRNA hybridizes to VSG-20 BC3 and also to two other loci as well. The cDNA probe specific for the $5^{\prime}$ end of VSG-20* mRNA hybridizes to a single locus, different from the VSG-20 and VSG-20bis BCs.

We cloned the three members of VSG-20bis gene family (VSG-20bis BCl, VSG-20bis, BC2 and VSG-20 $\mathrm{BC} 3$ ) and VSG-20* BC. The sequences of the cDNAs and BCs are presented in Figure 3.

\section{The VSG-20bis gene is a mosaic made with the $3^{\prime}$ donor of the VSG-20 gene}

The VSG-20bis gene is not the product of the duplication of a single $\mathrm{BC}$. Its sequence is very similar to the sequence of VSG-20 3' donor, VSG-20 BC3, that has been used as one of the templates. The sequence differences between the VSG-20bis ELC and VSG-20 BC3 are contained within a DNA segment of $356 \mathrm{bp}$. This $356-\mathrm{bp}$ DNA segment was most likely provided by another member of the VSG-20bis gene family, VSG-20bis BCl. Whereas neither VSG-20 BC3 nor VSG-20bis BCl has an open reading frame long enough to encode a VSG of the usual size range, the combination of the two BCs gen-
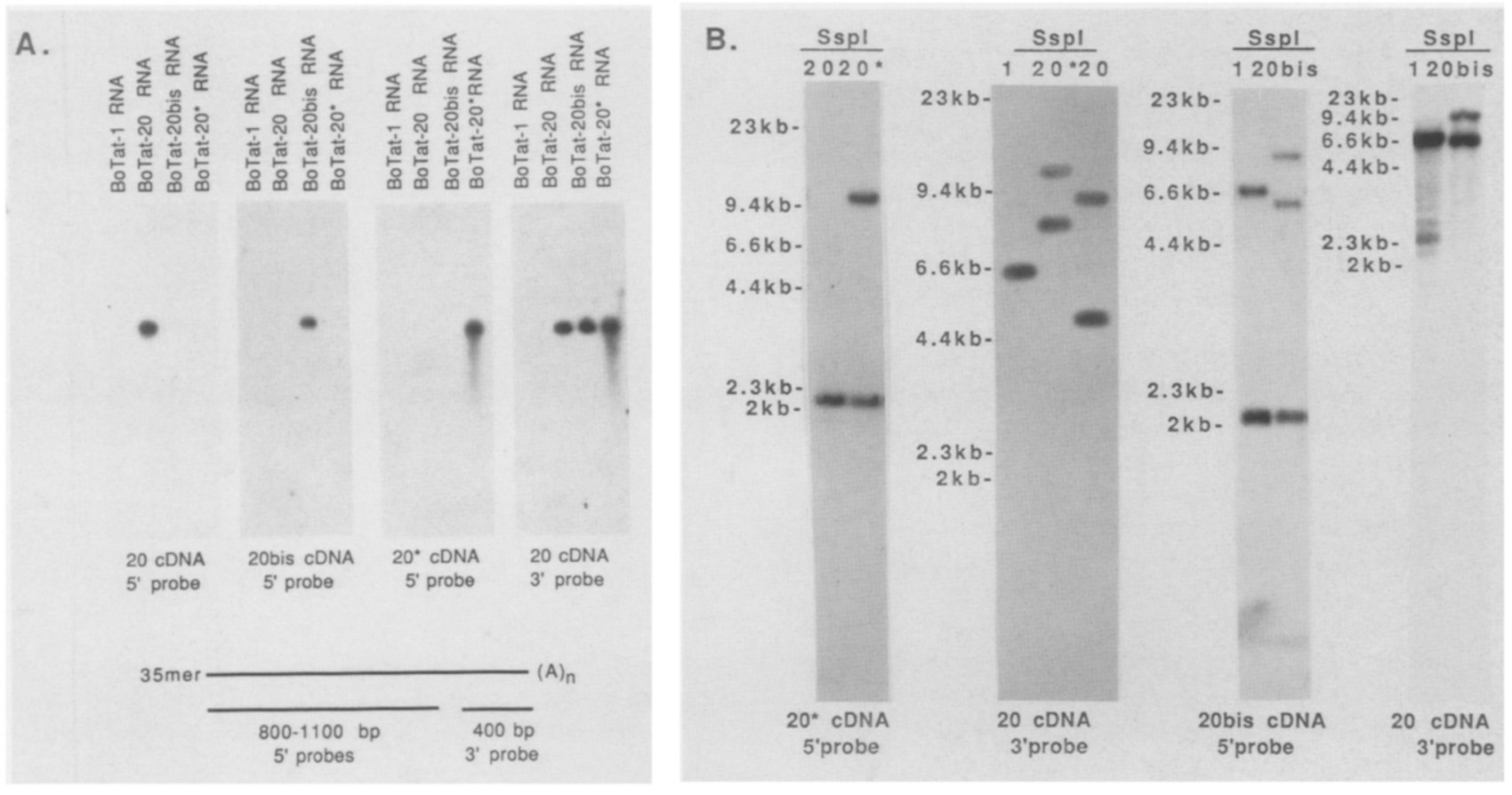

Figure 2. Composition of VSG-20bis and VSG-20^ gene families. (A) Probes complementary to the 5' portions of VSG-20, VSG-20bis, or VSG-20* mRNA, or $5^{\prime}$ probes, and a probe complementary to the $3^{\prime}$ portion of VSG-20 mRNA, or $3^{\prime}$ probe, were used on Northern blots. The $3^{\prime}$ probe hybridizes to an abundant transcript of the size of the VSG mRNA in the three variants. The $5^{\prime}$ probes hybridize to a transcript present only in the variant from which the probe was obtained. (B) The VSG-20 3' probe and VSG-20* or VSG-20bis $5^{\prime}$ probes were used on Southern blots. An ELC is present in each variant, which hybridizes specifically to the $5^{\prime}$ probe obtained from that variant. Both ELCs also hybridize to VSG-20 3' probe. Three genes other than VSG-20bis ELC hybridize to VSG-20bis 5' probe, one being VSG-20 BC3. The band at $2.1 \mathrm{~kb}$ is a doublet (see Experimental procedures). One gene other than VSG-20* ELC hybridizes to VSG-20* $5^{\prime}$ probe. 
A 20bis CDNA 20bis BC2 200is $B$ (2) $20 \mathrm{BC} 3$ GCCAGCAAATGTTTCGTGAaGCTTCCATAATCGTCCTTITGCTCCATTTGTGCTACAGGCAG CAGCATCTCACATITTATGGTGCTATAGTTAGACCGTCAAGT-TCAGCAAATGT TTCG

AGCCAGGCCTCGCACGCAGCCCTCGACGAAGCAAAAGCAACAGGACTCTGTAAAGTGGCAGCGGAACTATTAAAAGCCCCCGGCCTTGCGCTGCAAAAATACTCCGACTTGCAAGCG

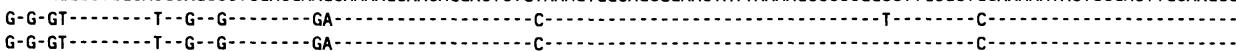
(1)

CGGGCAGCTGCCGCTGCAGAAGCGGCTTIACTGGCGTCGCTTGCAAGTGACCTGGAAAAGTCAATCAACATATCCACAGTITTCAGGGCGATAGCGCTATCTGCTGAGAAATGCGCA -

cGCAGATCGCTCGGCGAACTTAATTTGCTGGCTCAAAAGGCGACGAAaGCGACAGTAAACGCAGCGAAAGCGGCCGGCCACATAAGCGAGCTACTAGATTTACTACGCCAGGGATCA GT -GACG-T-AGT -GACG-T-A-A

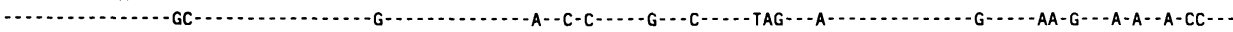

TACAACAGCGCAGTCCGTACCAAATGGTGCCTAGGATCGGCAGAAAACCCAACAGGAGCGGCGACTATAACGGCCTTAAGT TGCCCAACAGAGTACATAGACACTATAGCGCCAACG (1)

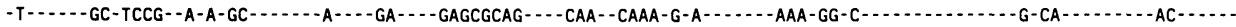
GCTAAGCTAGACGAACACATTATATCGGACACCGGCTACGCCGGTCTGTCTCCAGGAAGCGCGAAACTGAGCGGTACCGGGGGGCAAGCTGCGGACTACTAATITCAGCAACCAAC 0
0 A - A - ACCGG-G- - A GgACCAACAAACCTATGGATGACGACCGACCAGgCCAACATCAACGCTATAGCCGGTTTCATGACGCTGACAGCAAAGGACAACGCCGGTAGCGAGGACTACACAATCAACAGCGTG ACG-1... G

AAAACAGGCGCAAGCGCAGGGGCTTTTGGCAACCCCACAAACACGCCTCAAAAGATATACAACGACATAAAACAATTGGAAAAGCATACTGTCTCAGGCTGCGGGAACGACGTCGAC -

ACGGTACTGAAAACCGCGGTAAAGGAAAATACCGCGGCCAATCTACTCGAAGAACTTCTGAACACACAAGAACCATACAAAACCCAAAAAAATGCCGCACAAGCAGCCAAAGACATC

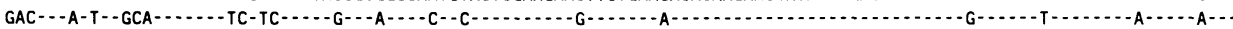

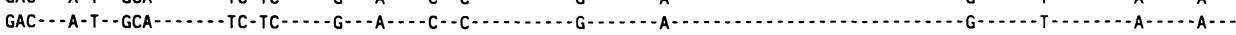

ATCAAACAAGCCGCGGACACCGACGgCCCCGACCAAGAGGGCGCGATCGTTAGAAAAATAAGAAGCCAAACGGCTGAGAGAATACAACAAGGCAAAATAGTGGGCAAGAAACTAAGT

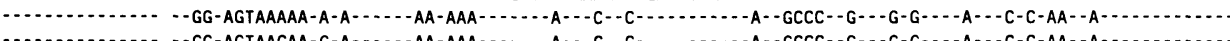

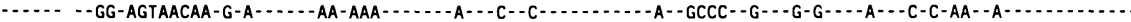

GACGACGCCACAGCAGACGAAAACATGCGCACTCTTC TA CTAAATCATCTAAAAAA CAGAGAACAATTAGACAAACTCGCTTCTGACATTGCGGCAGCAAAGGACACAGCAGCG

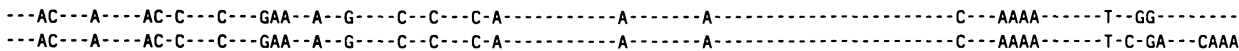

AAGGCGCCCCATGTACCAAAACCAGATGATTGCAAGGGTAAAAAGGGGGCTGACTGCAAAGATGGATGCGAGCTGGTAGAGGGGGTTTGCAAACCAGTAAAGCAAGGAGAGGGAGAA - - A - A -

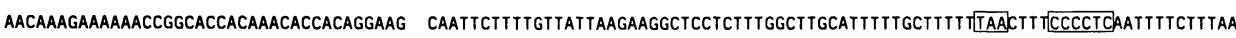
T--.-GCCCCC-CAA-CAGAGGG-GGAGAG-A--AT--AAA--C-GGAACCAC-AAC-CC-CAGGGAA-AA-TATA-TATAA--ACTCCCCCAAGT- - GATT-ATTT-C---G-ATT--.-AC-C--CAA-CAGAGGG-GGAGAG-A-- AAA--C-GGAACCAC-AAC-CC-CAGGGAA-AA-TATG-TGTAA--ACGCCCCCAGTG--GATT-ATTT-C- -TG-AT-

GGAAATT TGCTACTTGAAAAACTTTOTGATATATTTTAACACCT 3'

AT--ACG-TTATGA-TTTGTT-AACT-T-C-A- 31

AT--ACG-TTATGA-TITGTT-AACT-T-C-A- 3

AT.................................................

B 20* CDNA CACTACCTCCACAACGAAGCAGCGCAGGCACCAGTCTGGTGGGGAGGCAAATTITTCAAAACGGCGGGCAACAATGCAGACATCGGGGCGGCAAGCTGGGCAGCGA $20 * \mathrm{BC}$

20 CDNA ACAA--GA--TAGCGAC-TGGCTCGC - -ACA-G-CGACAAAAAAGCAG-T--CCGC--C-GA--TA-ACTT - -TC-GCA- - C-AAATA-A-A-GC-AATA-G-...

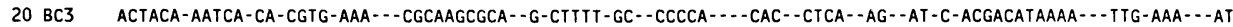

ATGACGAAGCCGACATCAGCAAAAGCAACTICCAAAAGTGCCACGAAACATTAAAGACAATACTCTCAAGCGGCGTCGACGACATGGCAACAAGCGCAAACTIAGCAAAGATACTA

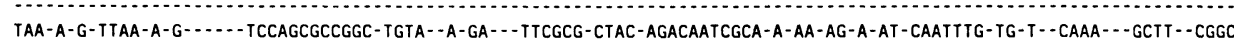
- CTGTCTCAGGCTGCGGGAACG-C-TCGACA-GGT-CTGAAA-- CGCGGTAA-GGA-A-TAC-G-GGCCAATCTACTCGAAGA-CTT-TGA-CA-A- - -GAACCAT-C-A-AC-C-

ACAAAAAATGCACCAGGCATAGCAGAACTAAACATCGCCAGCGGCAACATCCGAAAAAACGTCCCTTCAGCAGAAGCAAAACTAACACCGGCAGAGCTGACAACCCTGAGCAATGA - A- - C-G - - - GA - CGGGCCA - - -GTC - - AGAAC-GGAATTAGCGGCAGC - T - - CAAAATAC-AC-GTACC-A-GGGAC - -A-TT -AG - - C-AACTTTGGAA-GCA-T - .

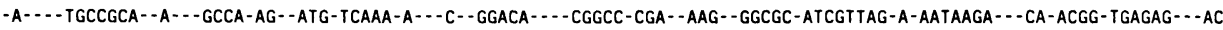

TATAGCATCTITGCCGAAAGTAACAGGAGCTGATCAGCTGAGCGATACGGAGCAAGATGCCAAGGACGTICAAGAAATTCTAGAGATCAACGAAACTGCCTGCAAGT TGGGGAAG A-G-ACGC-AGCTTACCTC-G-GATCAGAAAA-AG - - AA-C-ACA-AAATAG - A-ACTAG-AACAC-AAC - - -G- CGGT - AGC - - CA-CT -GG-G-CT - GTCAA-CAACT A-C-AGGCAAAATAGTGGGCA-GA-ACTAAGTGA-GA-GCCA-AGCAGACGAA- - - -.-GC-CTCTTC-ACTA- - TCATCTA-A-AAC-GAG---AATTA-ACAAACTCGCTICT

Figure 3. (Continued on following page.) 
Thon et al.

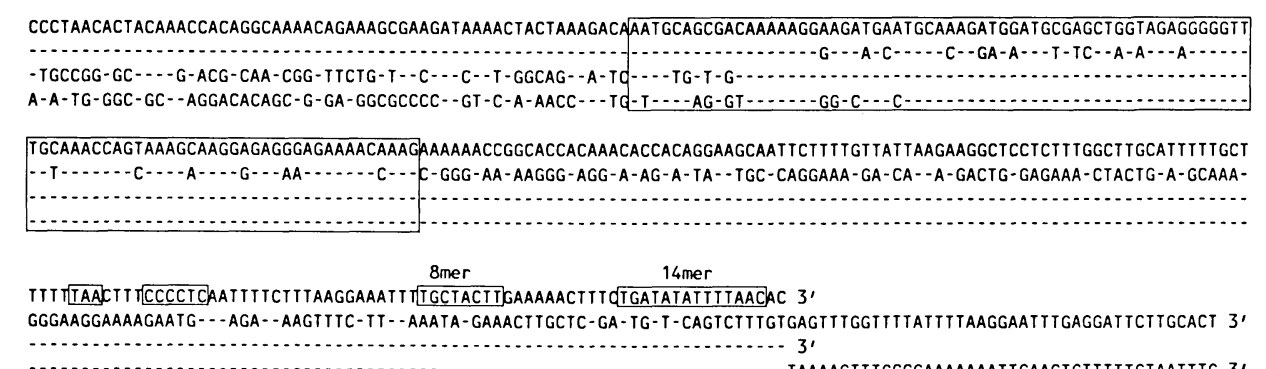
(3.

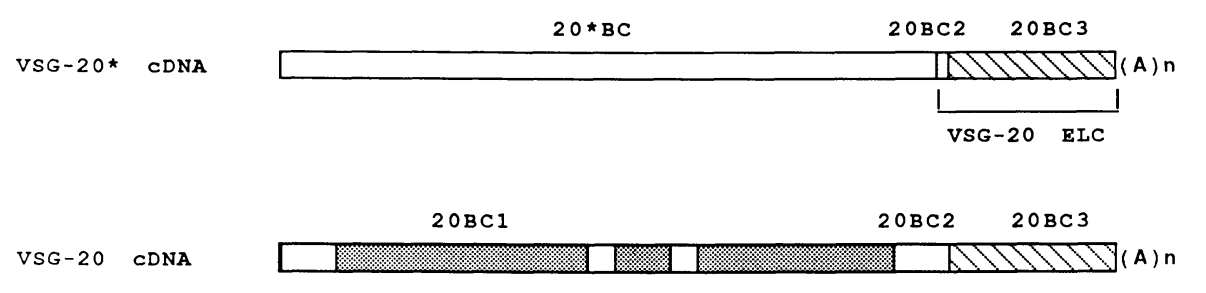

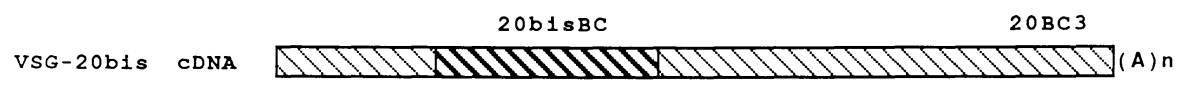

Figure 3. Sequence comparisons of VSG-20bis cDNA and VSG-20bis BCs $(A)$ and VSG-20* cDNA and VSG-20* BCs $(B)$.The sequences of VSG-20bis $(A)$ and VSG-20* $(B)$ cDNA are depicted and compared to the sequences of the BCs that hybridize to VSG-20bis and VSG-20* cDNA, respectively, on genomic Southern blots. The entire sequence of VSG-20bis cDNA is presented to show the contribution of the different BCs. As BoTat- $20^{*}$ was selected from BoTat-20, the sequence of VSG-20* gene is also compared with the sequence of VSG-20 gene. The sequence of only the most 3' 774 nucleotides of VSG-20* cDNA is shown. This segment contains the junction between VSG-20* BC and VSG-20 ELC in VSG-20* ELC. The entire sequence of VSG-20* cDNA is identical to the sequence of VSG-20* BC, $5^{\prime}$ of the junction. Dots in the BCs sequences stand for nucleotides that are identical to the nucleotide at the same position in the cDNA. (Bottom) The simplest combination of the donor genes that account for the cDNA sequences. The diagram for VSG-20 cDNA is from Thon et al. (1989).

erates an open reading frame of 477 amino acids. VSG-20bis BC1 fails to encode either of the conserved amino acid sequences found at the carboxyl terminus of VSGs (Boothroyd et al. 1981; Majumder et al. 1981; Matthyssens et al. 1981; Rice-Ficht et al. 1981; Liu et al. 1983 ) or the conserved sequences present in the $3^{\prime}$-untranslated region of VSG mRNAs (Liu et al. 1983). We cannot rule out that the third member of the VSG-20bis gene family, VSG-20bis $\mathrm{BC} 2$, which also contributed to the formation of VSG-20bis ELC. The sequence of VSG-20bis BC2 is closely related to the sequence of VSG-20bis $\mathrm{BCl}$ and also fails to encode a complete VSG. A contribution of VSG-20bis BC2 is not required to account for the sequence of VSG-20bis ELC.

The VSG-20* gene is a hybrid gene with the same $3^{\prime}$ end as the VSG-20 gene

BoTat-20* ELC is derived from BoTat-20 ELC. Two hundred ninety nucleotides at the $3^{\prime}$ end of the VSG-20 gene are retained in the new ELC, but all of the $5^{\prime}$ portion of the VSG-20 gene has been replaced by an unrelated sequence. This unrelated sequence is duplicated from VSG-20* $\mathrm{BC}$, which has a long open reading frame but none of the conserved features of the $3^{\prime}$ end of VSG genes. We noticed previously that the combination be- tween $5^{\prime}$ and $3^{\prime}$ donor genes in the VSG-20 ELC occurs within a short region in which the donors have related sequences (Thon et al. 1989). VSG-20* $\mathrm{BC}$ also is joined to the $3^{\prime}$ end of VSG-20 ELC within a very short region of similarity between the two sequences. The hybrid junction between $3^{\prime}$ and $5^{\prime}$ donor is found at approximately the same position in BoTat-20 and BoTat-20* ELCs.

\section{Detection of antigenic variants by polymerase chain reaction}

The characterization of three VSG $5^{\prime}$ donors, that can be expressed when recombined with VSG-20 BC3 as a $3^{\prime}$ donor, allowed us to test our model for the order of expression of the VSGs (Fig. 1). BoTat-20^ was selected from a population derived from a cloned BoTat -20 . We asked whether populations derived from other cloned $T$. equiperdum variants, which appear homogenous by immunofluorescence tests, also contained small numbers of different antigenic variants. The presence of such antigenic variants would mean that a switch occurred within the clonal population and would indicate which variant would be likely to be expressed next if the infection persisted under immunological selection. If the presence of sequence similarity between a $\mathrm{BC}$ and the ELC increases the probability of transposition of that $B C$ 
into the ELC, then switches to the expression of VSG-20 and VSG-20* should be observed more frequently in BoTat-20, BoTat-20*, and BoTat-20bis than in other serotypes. Furthermore, a sequence comparison of the BCs used in the generation of VSG-20, VSG-20*, and VSG-20bis genes (Fig. 4A) predicts that a switch from VSG-20 or VSG-20* to VSG-20bis is less likely to occur than the reverse because 20bisBC has very little homology with the BoTat- 20 or $20^{*}$ ELCs. This is shown schematically in Figure 4B.

cDNA first strands were made on RNA extracted from the following $T$. equiperdum variants: BoTat-1, BoTat20, BoTat-20bis, BoTat-20*, BoTat-14, BoTat-28, and BoTat-78. Oligonucleotides, whose sequences are indicated in Figure 5, were used as primers for reverse transcription. The same oligonucleotides, or $3^{\prime}$ primers, were subsequently used in combination with $5^{\prime}$ primers to amplify the cDNA templates by polymerase chain reaction (PCR). Pairs of primers specific for VSG-1, VSG-20, VSG-20bis, VSG-20*, or VSG-28 genes were used, respectively, in such reactions. This approach revealed the presence of trypanosomes expressing genes related to the VSG-1 gene in all variants. The VSG-1-related transcripts could be derived either from a low level of transcription of the VSG-1 BCs or from a small number of variants expressing VSG-1 in each population. This last possibility seems likely as variants expressing VSG-1 can be selected in the mouse after infection by BoTat-20 (Table 1). None of the VSG-20, VSG-20*, or VSG-20bis sequences were found in the amplified products of any unrelated variant. However, VSG-20 and VSG-20* sequences were found in amplified products of BoTat-20, BoTat- $20^{*}$, and BoTat-20bis. On the other hand, BoTat-20bis sequences were only found in amplified products of BoTat-20bis and not in those of either BoTat-20 or BoTat-20*.

To verify that similar numbers of trypanosomes were represented in each PCR reaction, we diluted the RNA preparations of the seven variants examined and used decreasing amounts of RNA for the first-strand cDNA synthesis. Identical volume fractions of the reverse transcription reactions were subsequently amplified with the VSG specific primers (14-merP and 35-merP in Fig. 5 ). The signal generated by the amplified material was lost at identical dilutions for all RNA preparations, indicating that each PCR reaction was representative of a similar number of trypanosomes. An analogous experiment is presented in Figure 5. BoTat-20bis RNA was mixed in various proportions with either BoTat- 20 or BoTat- $20^{\star}$ RNA, the total amount of RNA in the reaction being kept constant. PCR amplification of the first strand synthesized on the mixed RNAs shows that one BoTat-20bis variant could be detected in $10^{9}$ trypanosomes.

\section{Discussion}

During infections of rabbits, T. equiperdum variants have been shown to appear in a loose order (Capbern et al. 1977), in such a way that the different serotypes can be classified as early, middle, or late. There is also evidence that VSGs of the related species T. brucei are similarly expressed in an ordered manner, although extensive analysis has not been performed (Gray 1965; Lee et al. 1987; Timmers et al. 1987). This timing of antigen expression is poorly understood and may reflect multiple factors. For example, differences in growth rates and competition between variants may have an effect (Seed et al. 1984). Also, the transient expression by a single cell of two different antigens at a time, which has been shown to occur in culture (Baltz et al. 1986), may be a step in the antigenic switch. Thus, the ability to be coexpressed and maintained at the cell surface may also determine that VSGs can be successively expressed. Our finding that the late VSGs of $T$. equiperdum are encoded by composite genes constructed from pseudogenes suggested the model presented in the introduction.

Our model for ordered VSG gene expression makes several predictions that we have tested. First, because they are complete with respect to open reading frame and have homology with expression sites outside of their open reading frame, BCs encoding early VSGs should transpose in all variants with similar high probabilities. We verified that this is the case for the predominant early antigenic type, BoTat-1. Switches to the expression of VSG-1 gene could be detected in all clones examined, whether they were early (BoTat-14), or late (BoTat-28, BoTat-78, BoTat-20, BoTat-20*, BoTat-20bis).

Another prediction of our model is that the possibility of transposition of late BCs should depend on their having sequence homology with the gene occupying the expression site. In a variant that expresses an unrelated VSG gene, this transposition rate should be significantly lower than the rate of an early BC. As predicted, the expression of VSG-28 was not detected in BoTat-1, BoTat-14, BoTat-78, BoTat-20, BoTat-20*, or BoTat-20bis, and the expression of VSG-20, VSG-20* and VSG-20bis was not detected in BoTat-1, BoTat-14, BoTat-28, or BoTat-78.

A higher rate of transposition would be expected for the same late $\mathrm{BC}$ in a variant that expresses an ELC with which it has sequence similarity. Our approach allowed us to identify a set of BCs that share various lengths of sequence similarity. VSG-20, VSG-20*, and VSG-20bis BCs are all pseudogenes and can be expressed only as composite VSG genes. Although they are used in combination with the same $3^{\prime}$ donor, they encode VSGs that are seen as non-cross-reactive antigenic variants by their host. A replacement of one $\mathrm{BC}$ by another $\mathrm{BC}$ of the set in the expression site would therefore lead to a successful antigenic variation.

An 85-nucleotide sequence of VSG-20 and VSG-20* $5^{\prime}$ donors is highly related to a sequence in the BoTat-20bis ELC, donated by the VSG-20 BC3. VSG-20 and VSG-20* 5 ' donors might therefore productively recombine with VSG-20bis ELC. Our results show that such events occur at a detectable rate. Similarly, switches occur from the expression of VSG-20* to VSG-20, and vice versa. On the other hand, the $5^{\prime}$ donor of the VSG-20bis 
Thon et al.

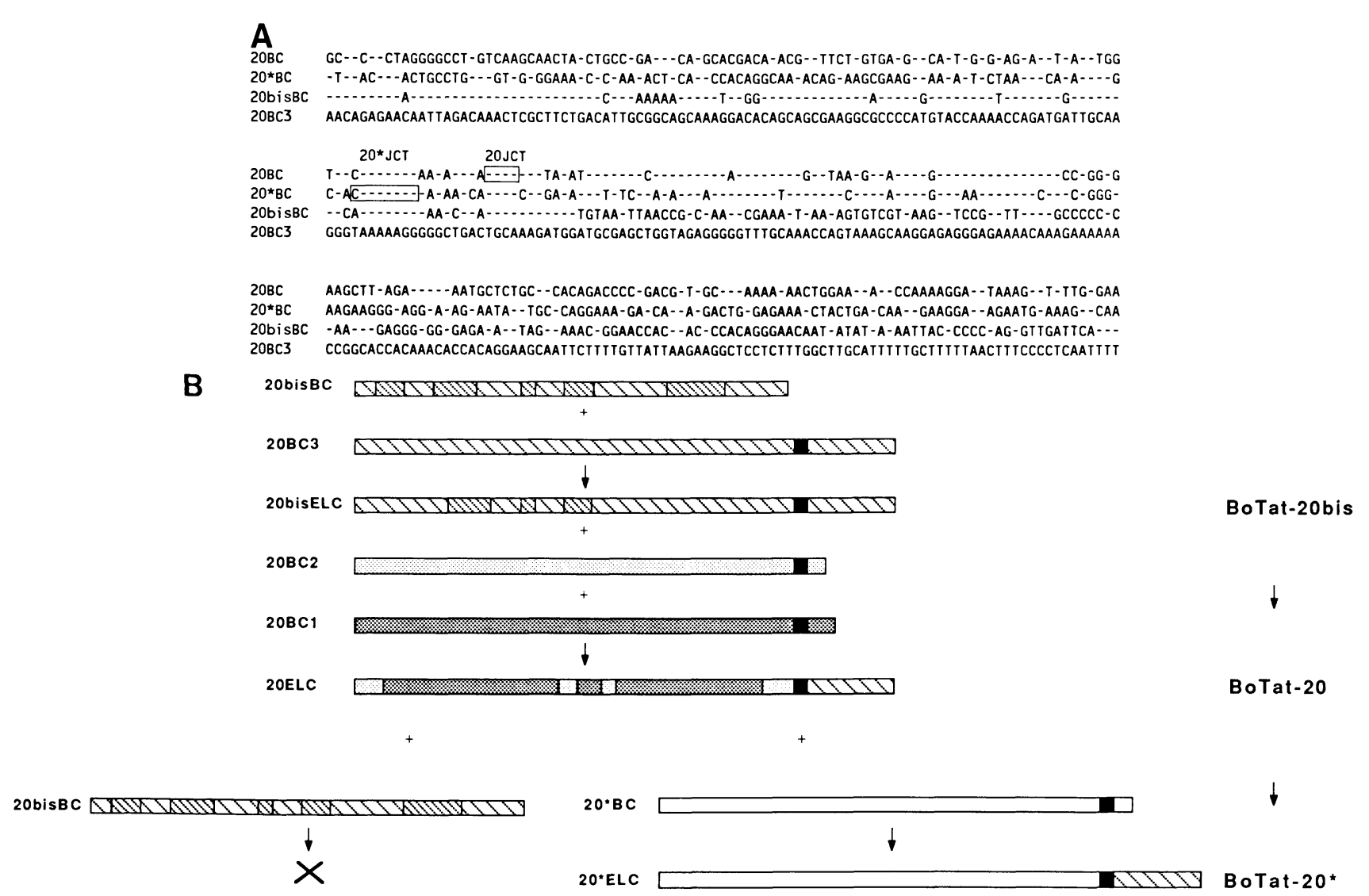

Figure 4. Comparison of VSG-20, VSG-20*, and VSG-20bis BCs. $(A)$ The sequences of the $5^{\prime}$ donor genes that contribute to the formation of VSG-20, VSG-20*, and VSG-20bis ELCs are compared to the sequence of the $3^{\prime}$ donor, 20BC3, around the point at which the recombination is found in the genes coding for VSG-20 and VSG-20*. Dashes indicate which nucleotides in their sequences are identical to the nucleotide occupying the homologous position in 20BC3. (20*JCT) Area in which the recombination occurs between $20^{\star} \mathrm{BC}$ and 20ELC; $(20 \mathrm{JCT})$ area in which the recombination occurs between $20 \mathrm{BC} 2$ and $20 \mathrm{BC} 3$. The sequence similarities between $20 \mathrm{BC} 3$ and $20 \mathrm{BC} 2$ or $20^{*} \mathrm{BC}$ span approximately the same gene segment. The sequence homology between $20 \mathrm{BC} 3$ and $20 \mathrm{bis} \mathrm{BC}$ is located $5^{\prime}$ of that segment, in such a way that there is only a short stretch of sequence similarity between 20 bisBC and $20 \mathrm{BC} 2$ or $20^{\star} \mathrm{BC}$. $(B)$ Diagram illustrating how the VSG-20 or the VSG-20* $5^{\prime}$ donors could productively recombine within VSG-20bis ELC or how they could replace each other in an expression site with a small region of sequence identity at their $3^{\prime}$ ends. VSG-20bis BC could recombine with VSG-20 BC3 within a large region of homology between the two genes but not with VSG-20 or VSG-20* ELCs, because it does not share enough sequence similarity with them. The region of sequence similarity between VSG-20 BC2, VSG-20* BC, and VSG-20 BC3 (Fig. 3) is represented by a black box. The regions of VSG-20bis BC and VSG-20 BC, which have related sequences, are represented by diagonal stripes.

gene displays very little sequence homology with either BoTat-20 or BoTat-20* ELCs. Thus, if sequence homology is important for insertion into an expression site, the VSG-20bis 5' BC would not be likely to recombine with them. We find indeed that $<10^{-9}$ trypanosomes expressing VSG-20bis are present in serotypes 20 or $20^{*}$.

Because it is likely that one $3^{\prime}$ segment is used with many $5^{\prime} \mathrm{BCs}$, the ultimate order of expression of the VSG genes must depend on the ordered insertion of $3^{\prime}$ donor genes into the expression sites. Although the two 3 ' donor silent genes that we have characterized thus far are pseudogenes (Roth et al. 1989; Thon et al. 1989), both are located at telomeres and are flanked on their $5^{\prime}$ side by barren regions, as are the silent copies of most complete early VSG genes. Thus, it is likely that these late 3 ' donors behave like early BCs and are inserted into expression sites with high probability. Because two ex- pression sites can be active in the same cells (Baltz et al. 1986), insertion of a 3' donor pseudogene into an expression site accompanied by activation of another site containing a complete gene would be possible and nonlethal to the cell. Subsequent gene conversions by related $5^{\prime}$ donor genes could eventually correct the defects in the pseudogene. Thus, the degree of defectiveness of the $3^{\prime}$ donor pseudogenes would determine the timing of their use in expression sites.

Another possible means of generating ordered use of the $3^{\prime}$ donor pseudogenes is through homologous recombination with previous residents of expression sites as we have described for the $5^{\prime}$ BCs. In this case, one could imagine that a 3 ' donor might have a short region of homology with an ELC. It could then enter the expression site through this region. If, $3^{\prime}$ of this, there was another region of homology with another family of $5^{\prime}$ donor genes, the passage from one set of VSGs to another could 
be effected. Furthermore, this process could be extended to give all of the ordered VSG gene expression observed.

Our results demonstrate that the combination of homologous recombination and selection against the reappearance of variants in the same animal can give rise to a predictably ordered sequence of gene expression.

\section{Experimental procedures}

\section{Trypanosomes}

The trypanosome strain used in this study was described by Capbern et al. (1977), as were the procedures used for the cloning and characterization of the antigenic variants.

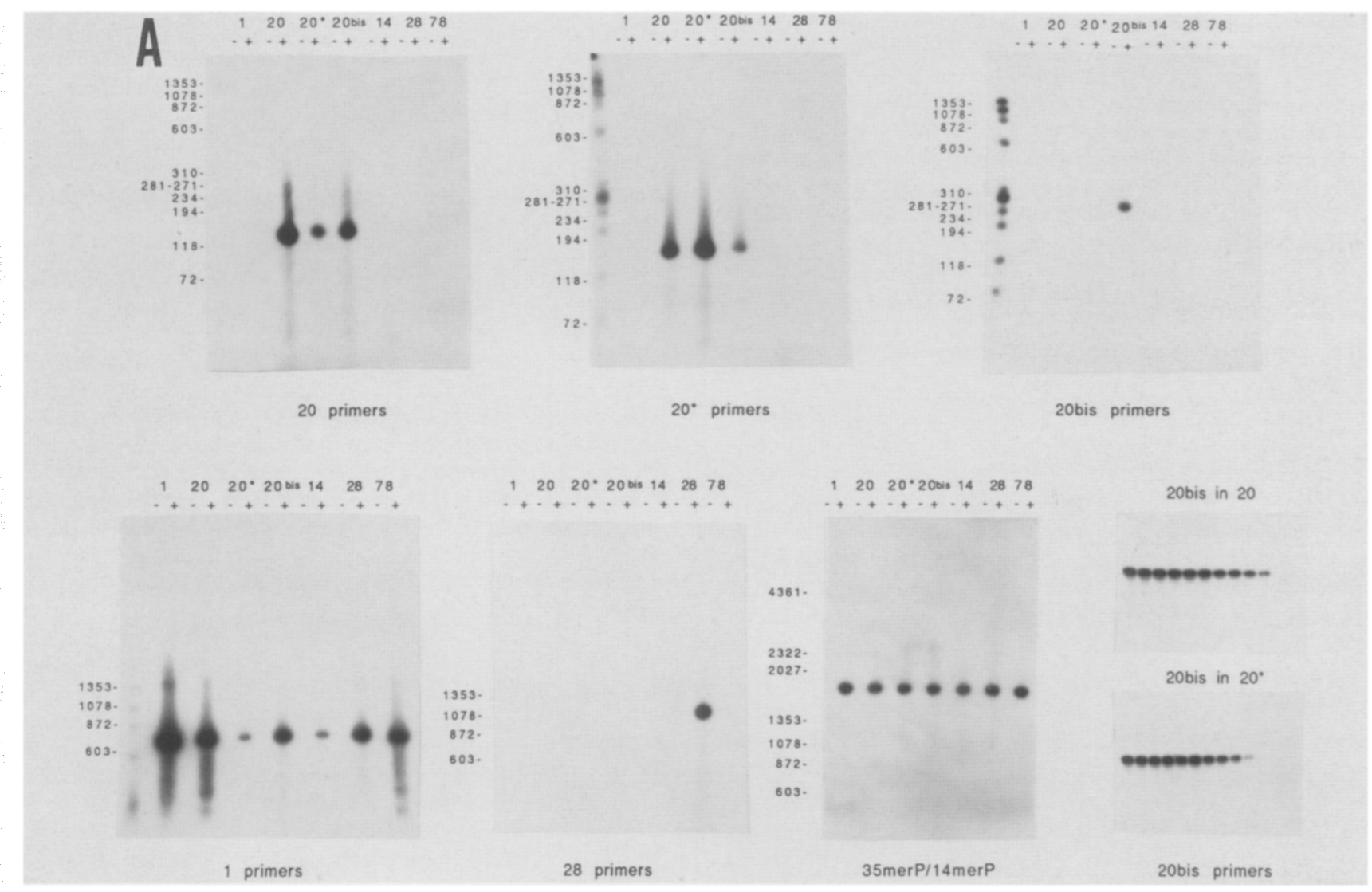

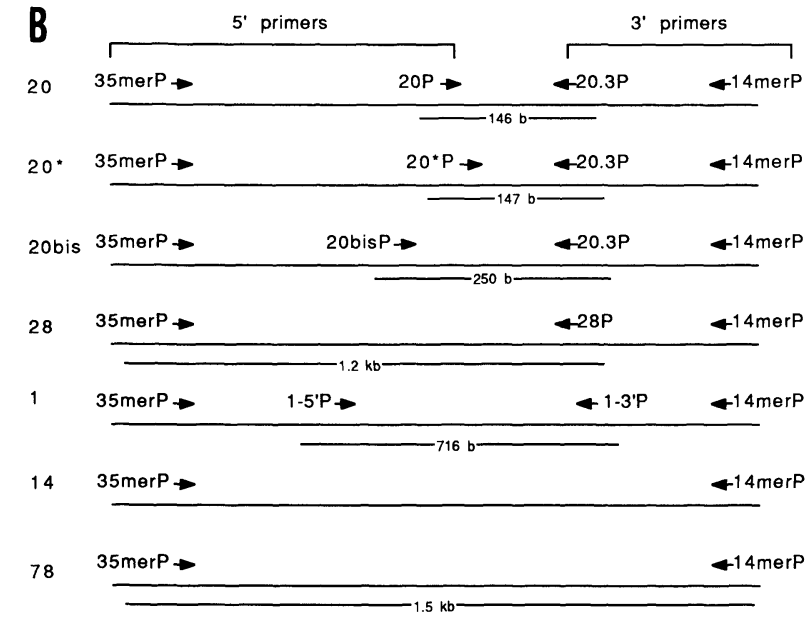

35merP: 5'AACGCTATTATTAGAACAG3'

2OP: 5'GGTGAAGCCACAGCTAG3'

20*P: 5'GATCAACGAAACTGC3'

20bisP: 5'GAAGCCAAACGGCTGAG3'

1-5'P: 5'GACACCAAGTGCGACCTAAACTCC3'
14merP: 5'GTGTTAAAATATATCAGAAAG3'

20.3P: 5'CTACCAGCTCGCATCC3'

28P: 5'GGGCATTCTGCTGGGG3'

1-3'P: 5'CGCCTTGTCACTCAATGTGCAC3'

Figure 5. Detection of antigenic variants by PCR. The presence of VSG-20-, VSG-20*-, VSG-20bis-, VSG-28-, and VSG-1-related transcripts was assayed by PCR as described in Experimental procedures $(A)$. The serotype of the trypanosomes from which the RNA was extracted for the cDNA synthesis is reported above each lane; $(-)$ Reverse transcriptase was omitted in the reverse transcription reaction; $(+)$ reverse transcriptase was present for the reverse transcription. The pairs of primers used for each set of PCRs are indicated under each autoradiograph. [20 primers (20P/20.3P)] Specific for VSG-20 transcripts; [20* primers $\left.\left(20^{\star} \mathrm{P} / 20.3 \mathrm{P}\right)\right]$ specific for VSG-20* transcripts; [20bis primers (20bis $\mathrm{P} / 20.3 \mathrm{P})$ ] specific for VSG-20bis transcripts; [28 primers $(35-\mathrm{mer} / 28 \mathrm{P})]$ specific for VSG-28 transcripts; [1 primers $\left.\left(1-5^{\prime} \mathrm{P} / 1-3^{\prime} \mathrm{P}\right)\right]$ specific for VSG-1 transcripts; (35-merP/14-merP) amplifies any VSG transcript. BoTat-20bis RNA was mixed with either BoTat-20 RNA (20bis in 20) or BoTat-20* RNA (20bis in 20*) in various proportions, prior to reverse transcription and amplification. (Lane 1) BoTat-20bis RNA only; (lane 2) BoTat-20bis RNA/other RNA $=10^{-1}$; (lane 3): $10^{-2}$, etc.; (lane 10) $10^{-9}$; (lane 11) 10-10. The sequences of the primers and the sizes expected for the amplification products are indicated $(B)$. 
Table 1. Mice protection by immunization

\begin{tabular}{lccc}
\hline & \multicolumn{3}{c}{ Immunization } \\
\cline { 2 - 4 } Challenge & BoTat-20 & BoTat-20bis & BoTat-20* \\
\hline BoTat-20 & $3 / 5$ & $5 / 5$ & $5 / 5$ \\
BoTat-20bis & $5 / 5$ & $0 / 5$ & $5 / 5$ \\
BoTat-20* & $5 / 5$ & $5 / 5$ & $0 / 5$ \\
\hline
\end{tabular}

Mice were immunized and subsequently challenged with trypanosomes of the indicated serotypes. The number of deaths per the number of mice challenged is reported for each experiment. Immunization against any given serotype conferred protection against that serotype specifically. The three mice that died after immunization and challenge with BoTat-20 died from BoTat-1 infections, because some trypanosomes in the BoTat-20 population switched from the expression of VSG-20 to that of VSG-1.

\section{Monoclonal antibodies}

Hybridomas producing anti-VSG-20 antibodies were produced as described by Esser et al. (1982).

\section{Mouse immunization and challenge}

At day $0,2 \times 10^{7}$ trypanosomes killed by being frozen and thawed were injected to each mouse in the presence of complete Freund's adjuvant. The same number of killed trypanosomes were injected without adjuvant at day 14. Live trypanosomes $\left(10^{3}\right)$ were injected at day 21 for the challenge.

\section{cDNA cloning}

BoTat-20bis cDNA BoTat-20bis RNA purified on oligo(dT) Sepharose and an Invitrogen cDNA cloning kit were used to construct a BoTat-20bis cDNA library. A probe complementary to VSG-20 mRNA 3' end was used to screen this library. Seventeen clones hybridizing to VSG-20 mRNA 3' probe were characterized further. These clones contain overlapping inserts ranging from 600 to $1500 \mathrm{bp}$. The inserts are complementary to a 1.5-kb abundant transcript whose expression is BoTat-20 specific. They hybridize to an ELC in BoTat-20bis genomic DNA. One of these clones was expressed in Escherichia coli and a rabbit serum raised against the expression product. The serum reacts with a $55-\mathrm{kD}$ protein present in BoTat-20bis and not in BoTat-1 (data not shown). These are properties expected for cDNA clones complementary to VSG-20bis mRNA.

BoTat-20* cDNA A cDNA first strand was made on BoTat$20^{*}$ mRNA with a primer complementary to a conserved sequence at the $3^{\prime}$ end of VSG mRNAs (14-merP in Fig. 5B). This first strand was amplified by PCR with the same $3^{\prime}$ primer as for the first-strand synthesis and a $5^{\prime}$ primer with part of the $T$. equiperdum 35-mer miniexon sequence (35-merP in Fig. 5B). The PCR conditions were as indicated below. The amplification generated a $1.5-\mathrm{kb}$ product stained on gels by ethidium bromide. The amplification products of two independent reactions were phosphorylated and cloned into the SmaI restriction site of plasmid vector Bluescribe(+) (Vector Cloning Systems). One clone derived from each amplification reaction was characterized. The inserts of the two clones are $1.5 \mathrm{~kb}$ long and have identical restriction maps. They hybridize to a BoTat- $20^{\star}$ specific transcript and to an ELC in BoTat-20* genomic DNA. The sequence of one strand was determined on a clone derived from one of the amplification reactions, and the sequence of the complementary strand was determined on a clone derived from the other amplification reaction. The two sequences are identical.

\section{Genomic cloning}

VSG-20bis BCs BoTat-20bis genomic DNA was digested with the restriction enzyme SspI and inserted into the SmaI restriction site of the plasmid Bluescribe/ + ) /Vector Cloning Systems). Twelve clones hybridizing to a probe complementary to the $5^{\prime}$ end of VSG-20bis mRNA were isolated and characterized. Each of these clones has a $2.1-\mathrm{kb}$ insert, and they can be classified into two groups by restriction mapping and partial sequence analysis. Restriction maps are as predicted from Southern blots of genomic DNA. A partial DNA sequence of a member of each group was determined (Fig. 3).

VSG-20 BC3 Part of VSG-20 BC3 had been cloned previously (Thon et al. 1989). We obtained an overlapping clone from BoTat-20bis DNA, which contains VSG-20 BC3 $5^{\prime}$ end. This clone, p5'20BC3, contains a 1.6-kb SspI-SalI restriction fragment inserted between the SmaI and SalI restriction sites of Bluescribe - - (Vector Cloning Systems). The restriction map of the insert is as expected for BC3. The overlap of this fragment with the HaeII restriction fragment cloned from BoTat-20 DNA (Thon et al. 1989) is $350 \mathrm{bp}$ long, and the sequence of that segment is the same in the two clones. The sequence presented in Figure 3 was obtained from p5'20BC3 for the most 5' $880 \mathrm{nu}-$ cleotides and from BoTat-20 genomic clones for the most 3' 600 nucleotides.

$V S G-20^{*} B C s$ A genomic library of BoTat $20^{*}$ DNA digested with the restriction enzyme $S s p$ I was made in the SmaI restriction site of the plasmid Bluescribe(+) (Vector Cloning Systems). Six clones hybridizing to a probe complementary to the $5^{\prime}$ end of VSG-20* mRNA were isolated and characterized. Each of these clones has a $2-\mathrm{kb}$ insert. The restriction map of the insert is the same in all clones and corresponds to the restriction map established from genomic Southern blots for VSG-20* BC.

Northern and Southern blotting Trypanosome RNA or genomic DNA digested with various restriction enzymes was fractionated in agarose gels and transferred to GeneScreen Plus nylon membranes (NEN Research Products). Hybridization to ${ }^{32} \mathrm{P}$-labeled cDNA probes was performed as suggested by the suppliers.

DNA sequencing cDNA and genomic clones were subcloned, and single-stranded templates were made in E. coli strain TG1 in the presence of helper phage M13K07. Sequencing reactions were performed on the single-stranded templates by use of a Sequenase DNA sequencing kit (U.S. Biochemicals) with either the provided M13 primer or specific 18-mer oligonucleotides synthesized on an Applied Biosystems DNA synthesizer. DNA sequences were compared by use of Genepro Sequence Analysis software (Riverside Scientific Enterprises).

cDNA synthesis and PCR The conditions used for the cDNA synthesis were as follow: Ten micrograms of total, DNasetreated, trypanosome RNA and $200 \mathrm{ng}$ of a specific oligonucleotide $\left(3^{\prime}\right.$ primer) were incubated in $20 \mu \mathrm{l}$ of $0.5 \mathrm{~mm}$ each dATP, dCTP, dGTP, and dTTP, $100 \mathrm{mM}$ Tris- $\mathrm{HCl}$ (pH 8.3), 50 $\mathrm{mM} \mathrm{KCl}, 10 \mathrm{~mm} \mathrm{MgCl}_{2}, 10 \mathrm{~mm} \mathrm{DTT}$, in the presence of RNasin, and 5 units of AMV reverse transcriptase (BRL) for 90 min at $42^{\circ} \mathrm{C}$. Two microliters of the cDNA synthesis reactions 
was subsequently added to $48 \mu \mathrm{l}$ of the following mixture: 50 $\mathrm{mm} \mathrm{KCl}, 6 \mathrm{~mm}$ Tris- $\mathrm{HCl}$ (pH 8.3) $1.5 \mathrm{~mm} \mathrm{MgCl}_{2}, 0.01 \%$ (wt/ vol) gelatin, $0.25 \mathrm{~mm}$ each dATP, dCTP, dGTP, and dTTP, 10 $\mu \mathrm{Ci}\left[\alpha^{-32} \mathrm{P}\right] \mathrm{dCTP}(800 \mathrm{Ci} / \mathrm{mmole}), 10 \mu \mathrm{M} 3^{\prime}$ primer, $10 \mu \mathrm{M} 5^{\prime}$ primer, and 1.25 units Taq polymerase (Perkin-Elmer Cetus). The amplification reactions were 30 cycles $\left(1 \mathrm{~min}\right.$ at $94^{\circ} \mathrm{C}_{;} 3$ $\min$ at $55^{\circ} \mathrm{C} ; 4$ min at $72^{\circ} \mathrm{C}$ ), performed in a Perkin-Elmer Cetus DNA thermal cycler. The amplification products $(10 \mu l$ of each PCR reaction) were size-fractionated either in $4 \%$ NuSieve agarose (FMC) gels for the small fragments (120-230 bp) or in $1 \%$ agarose (BRL) gels for the bigger fragments (1.2-1.5 kb), and the gels were autoradiographed.

\section{Acknowledgments}

We thank Robert Levis, Andrew Strand, and Andrew Taylor for their comments on this paper. This research was supported by grants from the Centre National de la Recherche Scientifique, and the Institut National de la Santé et de la Recherche Médicale, The National Science Foundation (grant DCB-8602729), and the American Cancer Society (grant NP-585).

The publication costs of this article were defrayed in part by payment of page charges. This article must therefore be hereby marked "advertisement" in accordance with 18 USC section 1734 solely to indicate this fact.

\section{References}

Baltz, T., C. Giroud, D. Baltz, C. Roth, A. Raibaud, and H. Eisen. 1986. Stable expression of two variable surface glycoproteins by cloned Trypanosoma equiperdum. Nature 319: 602-604.

Bernards, A., L.H.T. Van der Ploeg, A.C.C. Frasch, P. Borst, J.C. Boothroyd, S. Coleman, and G.A.M. Cross. 1981. Activation of trypanosome surface glycoprotein genes involves a duplication-transposition leading to an altered $3^{\prime}$ end. Cell 27: 497-505.

Boothroyd, J.C., C.A. Paynter, G.A.M. Cross, A. Bernards, and P. Borst. 1981. Variant surface glycoproteins of Trypanosoma brucei are synthetised with cleavable hydrophobic sequences at the carboxy and amino termini. Nucleic Acids Res. 9: 4735-4743.

Campbell D.A., M.P. Van Bree, and J.C. Boothroyd. 1984. The 5' limit of transposition and upstream barren region of a trypanosome VSG gene: tandem 76 base-pair repeats flanking

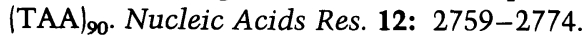

Capbern, A., C. Giroud, T. Baltz, and P. Mattern. 1977. Etude des variations antigenique au cours de la trypanosomie experimentale du Lapin. Exp. Parasitol. 42: 6-13.

De Lange, T., J.M. Kooter, P.A.M. Michels, and P. Borst. 1983. Telomere conversion in trypanosomes. Nucleic Acids Res. 11: 8149-8165.

Donelson, J.E., W.J. Murphy, S.T. Brentano, A.C. Rice-Ficht, and G.D. Cain. 1983. Comparison of the expression-linked extra copy (ELC) and basic copy $(\mathrm{BC})$ genes of a trypanosome surface antigen. J. Cell. Biochem. 23: 1-12.

Esser, K.M., M.J. Schoenbechler, and J.B. Gingrich. 1982. Trypanosoma rhodesiense blood forms express all antigen specificities relevant to protection against metacyclic (insect form) challenge. J. Immunol. 129: 1715-1717.

Florent, I., T. Baltz, A. Raibaud, and H. Eisen. 1987. On the role of repeated sequences $5^{\prime}$ to variable surface glycoprotein genes in African trypanosomes. Gene 53: 55-62.

Gray, A.R. 1965. Antigenic variation in a strain of Trypanosoma brucei transmitted by Glossina morsitans and G. palpalis. J. Gen. Microbiol. 41: 195-214.
Lee, M.G.S. and L.H.T. Van der Ploeg. 1987. Frequent independent duplicative transpositions activate a single VSG gene. Mol. Cell. Biol. 6: 357-364.

Liu, A.Y.C., L.H.T. Van der Ploeg, F.A.M. Rijsewijk, and P. Borst. 1983. The transcription unit of variant surface glycoprotein gene 118 of Trypanosoma brucei. J. Mol. Biol. 167: $57-75$.

Longacre, S. and H. Eisen. 1986. Expression of whole and hybrid genes in Trypanosoma equiperdum antigenic variation. $E M B O$ I. 5: 1057-1063.

Majumder, H.K., J.C. Boothroyd, and H. Weber. 1981. Homologous 3'-terminal regions of mRNAs for surface antigens of different antigenic variants of Trypanosoma brucei. Nucleic Acids Res. 9: 4745-4753.

Matthyssens, G., F. Michiels, R. Hamers, E. Pays, and M. Steinert. 1981. Two variant surface glycoproteins of Trypanosoma brucei have a conserved C-terminus. Nature 293: 230-233.

Michels, P.A.M., A.Y.C. Liu, A. Bernards, P. Sloof, M.M.W. Van der Bijl, A.H. Schinkel, H.H. Menke, P. Borst, G.H. Veeneman, M.C. Tromp, and J.H. Van Boom. 1983. Activation of the genes for variant surface glycoprotein 117 and 118 in Trypanosoma brucei. J. Mol. Biol. 166: 537-556.

Pays, E. and M. Steinert. 1988. Control of antigen gene expression in african trypanosomes. Annu. Rev. Genet. 22: 107126.

Pays, E., S. Van Assel, M. Laurent, B. Dero, F. Michiels, P. Kronenberger, G. Matthyssens, N. Van Merveinne, D. Le Ray, and M. Steinert. 1983. At least two transposed sequences are associated in the expression site of a surface antigen gene in different trypanosome clones. Cell 34: 359369.

Rice-Ficht, A.C., K.K. Chen, and J.E. Donelson. 1981. Sequence homologies near the C-termini of the variable surface glycoproteins of Trypanosoma brucei. Nature 294: 53-57.

Roth, C., S. Longacre, A. Raibaud, T. Baltz, and H. Eisen. 1986. The use of incomplete genes for the construction of a Trypanosoma equiperdum variant surface glycoprotein gene. EMBO J. 5: 1065-1070.

Roth, C., F. Bringaud, R.E. Layden, T. Baltz, and H. Eisen. 1989. Active late-appearing variable surface antigen genes in Trypanosoma equiperdum are constructed entirely from pseudogenes. Proc. Natl. Acad. Sci. USA 86: 9375-9379.

Seed, J.R., R. Edwards, and J. Sechelski. 1984. The ecology of antigenic variation. J. Protozool. 31: 48-53.

Thon, G., T. Baltz, and H. Eisen. 1989. Antigenic diversity by the recombination of pseudogenes. Genes Dev. 3: 12471254.

Timmers H.T.M., T. De Lange, J.M. Kooter, and P. Borst. 1987. Coincident multiple activations of the same surface antigen gene in Trypanosoma brucei. J. Mol. Biol. 194: 81-90. 


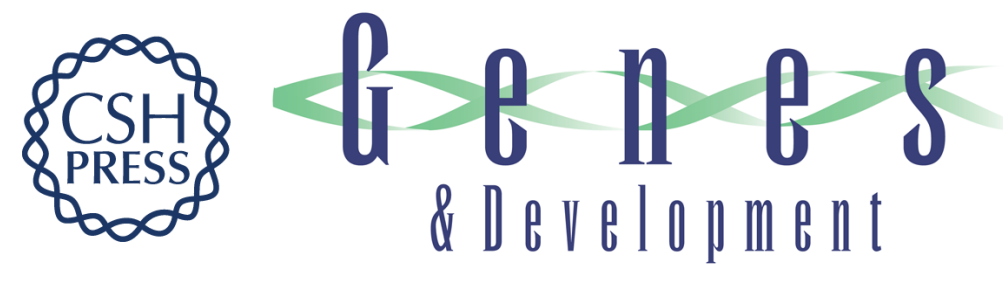

\section{Trypanosome variable surface glycoproteins: composite genes and order of expression.}

G Thon, T Baltz, C Giroud, et al.

Genes Dev. 1990, 4:

Access the most recent version at doi:10.1101/gad.4.8.1374

References This article cites 24 articles, 3 of which can be accessed free at: http://genesdev.cshlp.org/content/4/8/1374.full.html\#ref-list-1

License

Email Alerting

Receive free email alerts when new articles cite this article - sign up in the box at the top Service right corner of the article or click here.

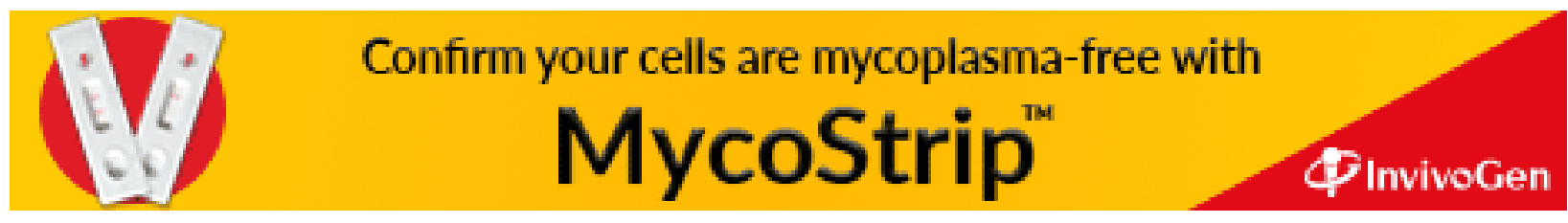

